\title{
On the spectrum of vertically propagating gravity waves generated by a transient heat source
}

\author{
M. J. Alexander ${ }^{1}$ and J. R. Holton ${ }^{2}$ \\ ${ }^{1}$ Colorado Research Associates Division, NorthWest Research Associates, Inc., Boulder, CO, USA \\ ${ }^{2}$ Department of Atmospheric Sciences, University of Washington, Seattle, WA, USA
}

Received: 13 October 2003 - Published in Atmos. Chem. Phys. Discuss.: 12 February 2004

Revised: - Accepted: 15 June 2004 - Published: 23 June 2004

\begin{abstract}
It is commonly believed that cumulus convection preferentially generates gravity waves with tropospheric vertical wavelengths approximately twice the depth of the convective heating. Individual cumulonimbus, however, act as short term transient heat sources (duration 10 to $30 \mathrm{~min}$ ). Gravity waves generated by such sources have broad frequency spectra and a wide range of vertical scales. The highfrequency components tend to have vertical wavelengths much greater than twice the depth of the heating. Such waves have large vertical group velocities, and are only observed for a short duration and at short horizontal distances from the convective source. At longer times and longer distances from the source the dominant wave components have short vertical wavelengths and much slower group velocities, and thus are more likely to be observed even though their contribution to the momentum flux in the upper stratosphere and mesosphere may be less than that of the high frequency waves. These properties of convectively generated waves are illustrated by a linear numerical model for the wave response to a specified transient heat source. The wave characteristics are documented through Fourier and Wavelet analysis, and implications for observing systems are discussed.
\end{abstract}

\section{Introduction}

Vertically propagating gravity waves generated by convection are known to provide an important contribution to the momentum budget of the middle atmosphere (see review by Fritts and Alexander (2003) and references therein). Several studies of gravity waves generated by thermal sources with vertical profiles resembling the positive half of a sine wave (e.g. Bretherton, 1988; Mapes, 1993; Nicholls et al., 1991; Pandya and Alexander, 1999) emphasize that the dominant

Correspondence to: J. Alexander

(alexand@cora.nwra. com) wave response occurs for waves with implied tropospheric vertical wavelengths of about twice the depth of the heating, in agreement with the fact that this is the wavelength that projects most strongly on the vertical heating profile. That this result is not critically dependent on the shape of the heating was shown by Salby and Garcia (1987), who found that for a wide variety of shapes of the heating profile the vertical scales of the response formed a narrow distribution in vertical wavenumber centered at about twice the effective vertical depth of the heating. Since the vertical wavelength is inversely proportional to the buoyancy frequency $N$, which in the stratosphere has approximately double its tropospheric value, vertical wavelengths observed in the stratosphere should, by the above argument, be about equal to the vertical depth of the tropospheric heating.

Observations from the Microwave Limb Sounder (MLS) on the Upper Atmosphere Research Satellite (UARS), however, suggest the existence of strong gravity wave variance in the stratosphere above convection with vertical wavelengths greater than $20 \mathrm{~km}$ (Alexander, 1998; McLandress et al., 2000). High frequency waves with vertical wavelengths in the range of $10-30 \mathrm{~km}$ are also believed to account for a significant share of gravity wave momentum flux in the mesosphere (Fritts and Vincent, 1987; Fritts and Alexander, 2003).

Some recent model studies also suggest that long vertical wavelength waves are important. In a study of gravity waves generated in a cloud resolving numerical model, Beres et al. (2002) showed that although the maximum gravity wave response in the stratosphere to numerically simulated tropical convection generally has a vertical wavelength about equal to the depth of the heating, there can be substantial power at vertical wavelengths substantially greater than the depth of the heating. This somewhat surprising result was discussed by Holton et al. (2002) who showed that for fixed frequency and fixed vertical depth of thermal forcing, the vertical wavelength of the maximum response to a specified heating depends strongly on the horizontal distribution of the heating, 
and that for parameters relevant to tropical convection the maximum response will generally be for stratospheric wavelengths that somewhat exceed the depth of the heating.

In the study of Holton et al. (2002) the heat source was localized in space but specified to be periodic in time. In reality cumulus clouds act as transient heat sources, and the frequency spectrum of the wave response can be quite broad. In this paper we utilize a simple numerical model to analyze the linear wave response to a transient heat source designed to emulate the heating by a deep cumulus cloud. Song et al. (2003) have pointed out that convection can generate gravity waves through nonlinear momentum and heat flux divergences and through latent heat release. In their analysis, the contributions of the nonlinear flux divergences and the diabatic heating were similar in magnitude, but tended to be out of phase. These authors found, consistent with the earlier analysis of Pandya and Alexander (1999), that utilizing the diabatic heating field from a nonlinear cloud model produces a field of gravity waves qualitatively in accord with the full nonlinear model, but with somewhat larger amplitudes. Thus, we can be confident that results from a linear model with a specified heating distribution provide a realistic qualitative approximation to gravity wave generation by cumulus convection.

\section{Linear wave equations for transient forcing}

The zonal and vertical momentum equations, continuity equation, and thermodynamic energy equation for twodimensional linear gravity waves excited by thermal forcing in an atmosphere at rest can be expressed in the Boussinesq approximation as

$\frac{\partial u^{\prime}}{\partial t}+\frac{1}{\rho_{0}} \frac{\partial p^{\prime}}{\partial x}=0$

$\frac{\partial w^{\prime}}{\partial t}+\frac{1}{\rho_{0}} \frac{\partial p^{\prime}}{\partial z}-b^{\prime}=0$

$\frac{\partial u^{\prime}}{\partial x}+\frac{\partial w^{\prime}}{\partial z}=0$

$\frac{\partial b^{\prime}}{\partial t}+w^{\prime} N^{2}=Q^{\prime}$

Here, $u^{\prime}, w^{\prime}$, and $p^{\prime}$ are zonal velocity, vertical velocity, and pressure disturbance fields; $b^{\prime} \equiv g \theta^{\prime} / \theta_{0}$ is the buoyancy, where $\theta^{\prime}$ and $\theta_{0}$ are the disturbance and basic state potential temperature, and $g$ is gravity; $Q^{\prime} \equiv g J^{\prime} / \theta_{0}$, where $J^{\prime}$ is the heating rate in $\mathrm{K} / \mathrm{s}$; and $\rho_{0}$ is a constant basic state value for density. The buoyancy frequency squared, $N^{2}$ is specified as

$N^{2}(z)=N_{0}^{2}\{2.5+1.5 \tanh [(z-2 h) / d]\}$ where $N_{0}^{2}=1 \times 10^{-4} \mathrm{~s}^{-2}, h=6 \mathrm{~km}, d=2 \mathrm{~km}$, and $z$ is expressed in $\mathrm{km}$. Equations (1)-(3) can be combined to eliminate $u^{\prime}$ and $p^{\prime}$ to get

$\frac{\partial}{\partial t}\left(\frac{\partial^{2} w^{\prime}}{\partial x^{2}}+\frac{\partial^{2} w^{\prime}}{\partial z^{2}}\right)=\frac{\partial^{2} b^{\prime}}{\partial x^{2}}$

Equations (4) and (6) can then be used to predict $w^{\prime}$ and $b^{\prime}$ for a known distribution of $Q^{\prime}$.

In order to mimic the behavior of a typical convective system we specify a transient heat source localized in time and space. The horizontal distribution is a Gaussian, the vertical distribution is a half-sine wave that propagates upward at a speed $c_{z}$, and the amplitude varies smoothly in time. The spatial and temporal dependence of the thermal forcing is given by:

$Q^{\prime}(x, z, t)=\hat{Q}(z, t) \exp \left[-\left(x-x_{0}\right)^{2} / \sigma_{x}^{2}\right]$

where

$\hat{Q}(z, t)= \begin{cases}Q_{0}(t) \sin \left[\frac{\pi}{h}\left(z-c_{z} t\right)\right] & 0 \leq z-c_{z} t \leq h \\ 0 & z-c_{z} t>h\end{cases}$

in which $Q_{0}(t)$ is specified as

$Q_{0}(t)=\left\{\begin{array}{l}\tau^{-1}[1-\cos (2 \pi t / \tau)] \\ 0 \quad 0 \leq t \leq \tau\end{array}\right.$

Thus, $\sigma_{x}$ is the horizontal scale of the forcing, $h$ is the vertical scale of the forcing at any instant, $h+c_{z} \tau$ is the total depth affected by the forcing, and $\tau$ is the total time that the forcing lasts. Note from Eq. (9) that the amplitude of the forcing is normalized by $\tau$ so the time integrated heating is independent of $\tau$.

If the $x$ dependence is expanded in a Fourier series then for each Fourier component the thermal source and the dependent variables can be expressed as

$$
\begin{array}{r}
Q^{\prime}(x, z, t)=\hat{Q}_{k}(z, t) \exp (i k x) \\
u^{\prime}(x, z, t)=\hat{u}_{k}(z, t) \exp (i k x) \\
w^{\prime}(x, z, t)=\hat{w}_{k}(z, t) \exp (i k x) \\
b^{\prime}(x, z, t)=\hat{b}_{k}(z, t) \exp (i k x)
\end{array}
$$

Where $k$ designates the zonal wavenumber. Substituting from (10) into (4) and (6) yields

$$
\begin{aligned}
& \frac{\partial}{\partial t}\left(\frac{\partial^{2} \hat{w}}{\partial z^{2}}-k^{2} \hat{w}\right)=-k^{2} \hat{b} \\
& \frac{\partial \hat{b}}{\partial t}+\hat{w} N^{2}=\hat{Q}
\end{aligned}
$$

Figure 1 shows the time-averaged heating and the buoyancy frequency as functions of height. 


\section{Numerical model}

A finite difference approximation to the system (11) and (12) is solved by using implicit time differencing and centered differencing in $z$. Once $\hat{w}$ is known the corresponding zonal velocity perturbation $\hat{u}$ can be computed from Eq. (3) by noting that

$i k \hat{u}=-\partial \hat{w} / \partial z$

and finite differencing the right hand side. In order to prevent reflection of waves from the top boundary at $150 \mathrm{~km}$, linear damping terms were added to Eqs. (11) and (12), with $e$-folding damping times increasing exponentially in height from a value of $5 \times 10^{4} \mathrm{~s}$ at the surface to $336 \mathrm{~s}$ at the top boundary. (Substantial damping of high frequency waves was limited to the region above about $80 \mathrm{~km}$ ).

Runs were carried out in a domain $2304 \mathrm{~km}$ wide and $150 \mathrm{~km}$ deep. 384 Fourier components were retained (corresponding to 768 grid points in $x$, with a grid spacing of $3 \mathrm{~km}$ ). The vertical grid spacing was specified to be $250 \mathrm{~m}$. The horizontal scale of the heat source was specified to be $\sigma_{x}=5 \mathrm{~km}$. The depth scale of the heating was $h=6 \mathrm{~km}$, and the level of maximum heating rose at a speed of $c_{z}=5 \mathrm{~m} \mathrm{~s}^{-1}$ so that the upper boundary of the heat source rose from 6 to $12 \mathrm{~km}$ during the $20 \mathrm{~min}$ period of forcing. The model was integrated forward for $8 \mathrm{~h}$, and the vertical velocity field was saved every 2 min. Figure 2 shows longitude-height cross sections of the vertical velocity field at hours $1,2,3$, and 4 . At hour 1 the flow is characterized by high frequency, long vertical wavelength wavepackets that propagate to the mesosphere close to the horizontal location of the heat source. At later times the field is progressively dominated by lower frequency and shorter vertical wavelength waves, which remain in the stratosphere while propagating horizontally far from the source. It is notable that the stratospheric wave response persists for several hours after the thermal forcing has diminished to zero.

\section{A simple wave analysis}

The zonal wavenumber dependence of vertical velocity amplitude, averaged vertically between 20 and $80 \mathrm{~km}$ is shown in Fig. 3. During the first $2 \mathrm{~h}$ the signal is dominated by waves in the horizontal wavelength range of $50-100 \mathrm{~km}$. These turn out to have very long vertical wavelengths (Fig. 4). At later times the signal separates into two wave packets with peaks at hour 3 near 100 and $30 \mathrm{~km}$, but shifting toward longer zonal wavelengths at later times. It is notable that amplitudes remain substantial even $6 \mathrm{~h}$ after cessation of the forcing.

Visualization of the time integration for each Fourier mode revealed that the characteristic vertical wavelength for each mode tended to decrease monotonically in time. Furthermore, the wavelength tended to be greater in the top half of
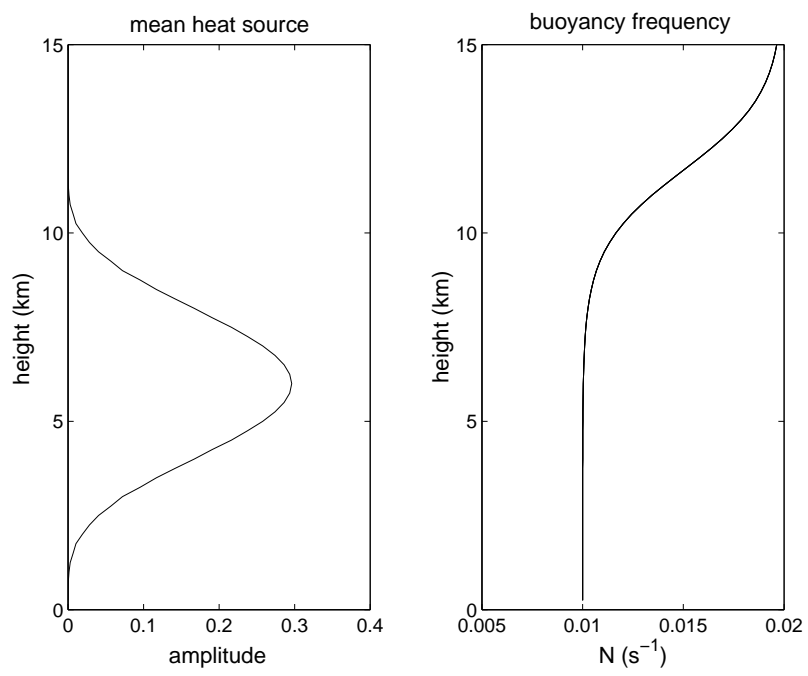

Fig. 1. The vertical profile of the time averaged forcing (left panel) and vertical profile of the buoyancy frequency (right panel) specified for the model.

the domain than in the bottom half. The dominant vertical wavelength in the middle part of the domain $(20-80 \mathrm{~km})$ was determined as a function of time by separately finding the distance in the vertical between nodes of the real and imaginary parts of each Fourier horizontal wavenumber component. These distances were doubled to give two estimates of the vertical wavelength, which were then averaged to give the final vertical wavelength estimate. In order to reduce noise in the calculation the wavelength estimates were averaged for 40 min periods.

Figure 4 shows the vertical wavelengths determined in the altitude range $20-80 \mathrm{~km}$ as a function of zonal wavenumber for averaging periods centered on hours 1, 2, 3, 4, and 6 of the simulation. Vertical wavelength decreases monotonically with increasing time. At low horizontal wavenumbers $(k)$, the average vertical wavelength also decreases with increasing $k$, although the very long vertical wavelengths diagnosed for zonal wavelengths longer than $\sim 200 \mathrm{~km}(k<.005)$ are of little significance because these waves have very small amplitudes (Fig. 3). According to the results in Fig. 4, only at the later times does the vertical wavelength over a significant range of horizontal wavenumbers approximate the often cited value of twice the depth of the heating times the ratio of the tropospheric buoyancy frequency to the stratospheric buoyancy frequency.

Once the average vertical wavelength is known for each horizontal wavenumber, it is possible to use the gravity wave dispersion relation to determine other averaged wave characteristics. Letting $L_{x}$ and $L_{z}$ denote the horizontal and vertical wavelengths, respectively, and focusing on the eastward propagating modes, the dispersion relation can be expressed 

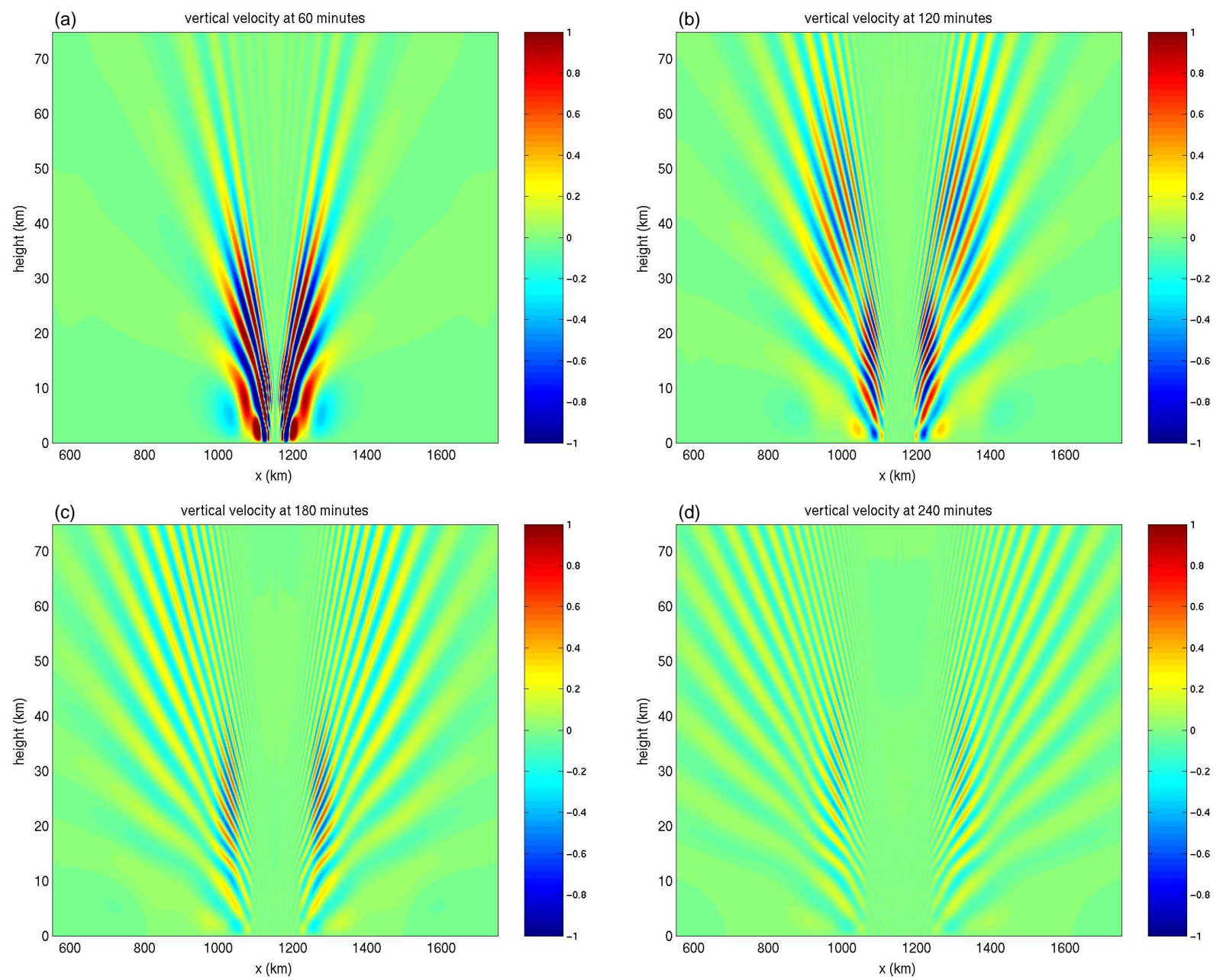

Fig. 2. Longitude-height cross sections of the vertical velocity field at hours 1, 2, 3, and 4.

in the form

$\omega=\frac{N}{\left[1+\left(L_{x} / L_{z}\right)^{2}\right]^{1 / 2}}$

where $\omega$ is the wave frequency and $N$ is the buoyancy frequency. The zonal phase speed, is then,

$c_{p x}=\frac{\omega}{k}=\frac{N L_{x}}{2 \pi\left[1+\left(L_{x} / L_{z}\right)^{2}\right]^{1 / 2}}$

For $L_{x} \gg L_{z}$ this expression simplifies to $c_{p x} \simeq N L_{z} /(2 \pi)$, which indicates that for most of the wavelength range of relevance here the zonal wavelength dependence of horizontal phase speed should have a similar structure to the profiles of vertical wavelength of Fig. 4.

The propagation of wave activity is determined by the group velocity,

$c_{g}=\left(c_{g x}, c_{g z}\right)=\frac{c_{p x}\left(L_{x} / L_{z}\right)}{1+\left(L_{x} / L_{z}\right)^{2}}\left(\frac{L_{x}}{L_{z}}, 1\right)$
Thus, $c_{g z} / c_{g x}=L_{z} / L_{x}$, so that group velocity is parallel to lines of constant phase. Further, for $L_{x} \gg L_{z}$ (16) shows that $c_{g x} \simeq c_{p x}$ and $c_{g z} \simeq c_{p x}\left(L_{z} / L_{x}\right)$.

The temporal evolutions of the frequency, vertical group velocity, and horizontal phase speed are shown as functions of the zonal wavenumber in Figs. 5-7, respectively. For all of the parameters displayed in Figs. 3-7 there is a strong time dependence so that application of conventional Fourier analysis to derive frequency and phase speed information must be done with care.

\section{Fourier Analysis}

Although the transient source in this study does not generate stationary time series, insight can still be gained from Fourier analysis in space and time. Figure 8 shows the results of the two-dimensional $(x, t)$ Fourier analysis for the cospectrum of $u^{\prime}$ and $w^{\prime}$ (e.g. Alexander and Holton, 1997) in the domain $0<t<8 \mathrm{~h}$ and $0<x<1150.5 \mathrm{~km}$ and averaged over the height range $14.25<z<75.25 \mathrm{~km}$. This analysis shows that 


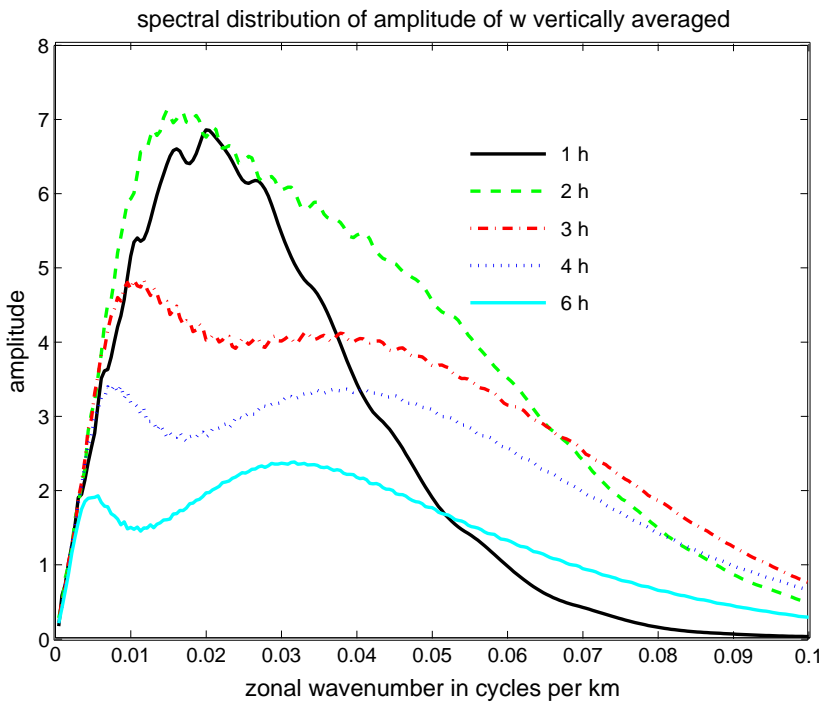

Fig. 3. Vertical velocity amplitude, averaged vertically between 20 and $80 \mathrm{~km}$, as a function of zonal wavenumber for 40 min averaging intervals centered on hours $1,2,3,4$, and 6 .

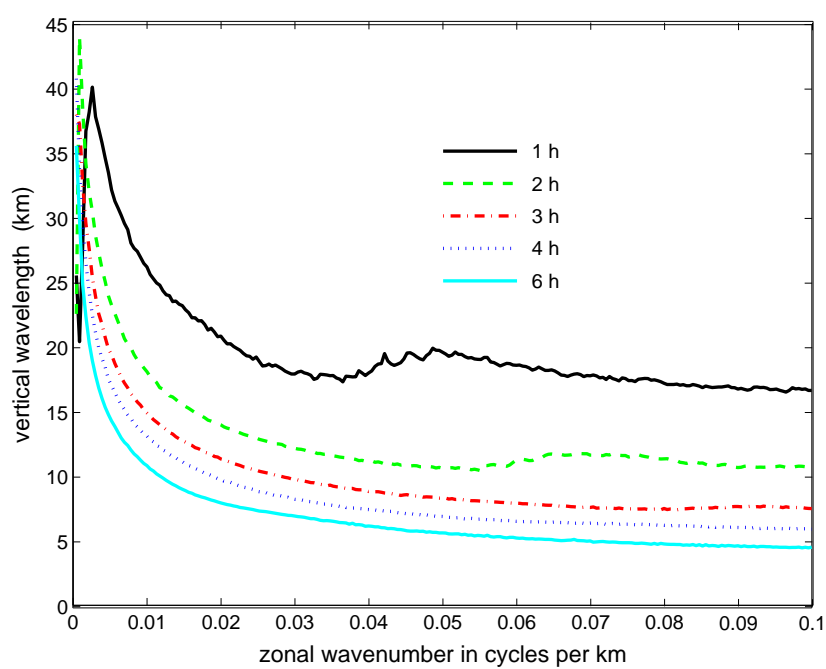

Fig. 4. Average vertical wavelength in the altitude range $20-80 \mathrm{~km}$ as a function of zonal wavenumber for $40 \mathrm{~min}$ time intervals centered on hours $1,2,3,4$, and 6 .

the waves are concentrated in two packets of wave activity with distinct vertical wavelengths of $\sim 4.3$ and $11 \mathrm{~km}$ that travel at horizontal phase speeds of about 15 and $40 \mathrm{~m} \mathrm{~s}^{-1}$, respectively, and hence are observed at different times at differing distances from the source. A careful examination of the panels of Fig. 2 confirms that the wave activity indeed is primarily associated with a fast moving wave packet followed by a slow moving packet.

Owing to the fact that except for the effect of variation in $N$ the horizontal component of group velocity is independent of height, above about $15 \mathrm{~km}$ all rays excited by

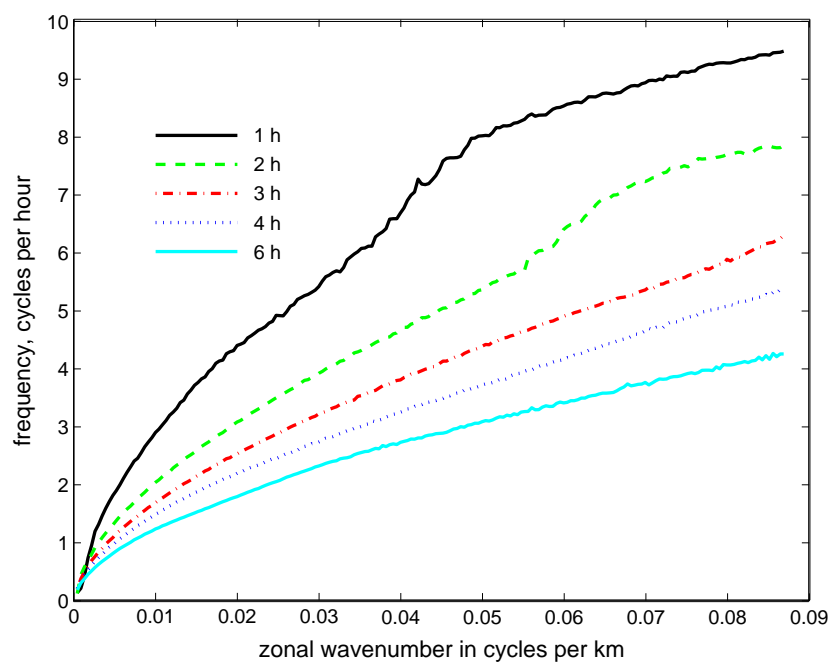

Fig. 5. Average frequency in the altitude range $20-80 \mathrm{~km}$ as a function of zonal wavenumber for $40 \mathrm{~min}$ time intervals centered on hours $1,2,3,4$, and 6 .

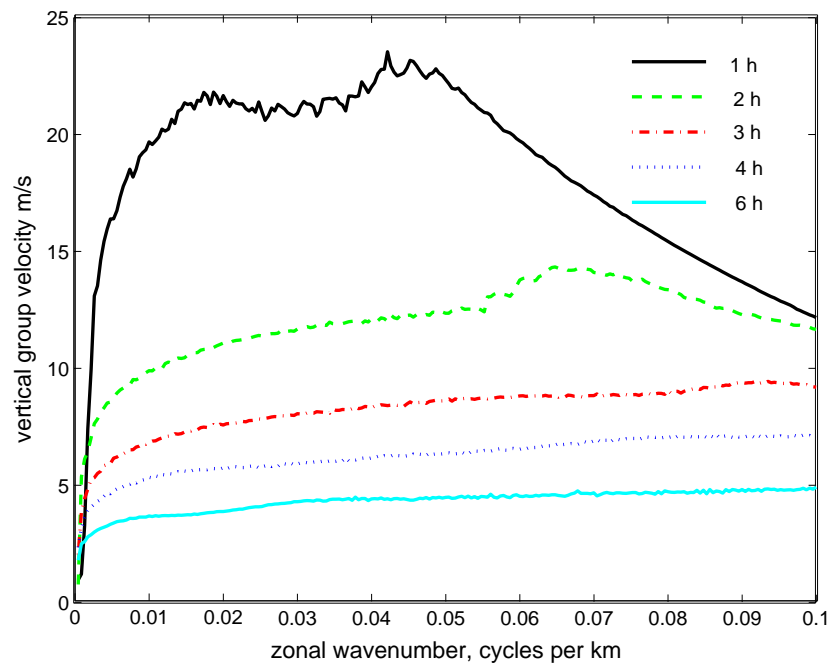

Fig. 6. Average vertical group velocity in the altitude range 20$80 \mathrm{~km}$ as a function of zonal wavenumber for $40 \mathrm{~min}$ time intervals centered on hours $1,2,3,4$, and 6 .

the forcing propagate horizontally away from the forcing region at a constant speed given by $c_{g x}$. For a given $L_{z} \ll L_{x}$, $c_{g x} \simeq c_{p x} \simeq N L_{z} / 2 \pi$. Hence the resulting pattern of wave activity in the stratosphere seen in Fig. 2 consists of two vertically aligned wave packets with different $L_{z}$, localized in space and traveling in the positive and negative $x$ directions, but with different speeds reflecting the distribution of vertical wavenumber, as shown in Fig. 8.

The preference for two fairly distinct vertical wavelengths $\sim 4.3$ and $11 \mathrm{~km}$ can be understood by noting that when the time averaged vertical profile of heating shown 


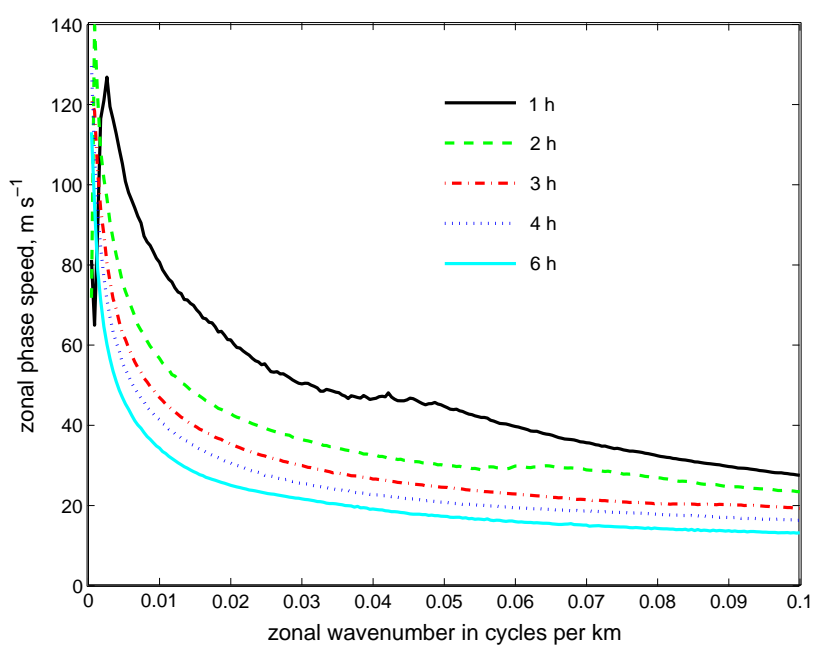

Fig. 7. Average horizontal phase speed in the altitude range 20$80 \mathrm{~km}$ as a function of zonal wavenumber for $40 \mathrm{~min}$ time intervals centered on hours $1,2,3,4$, and 6 .

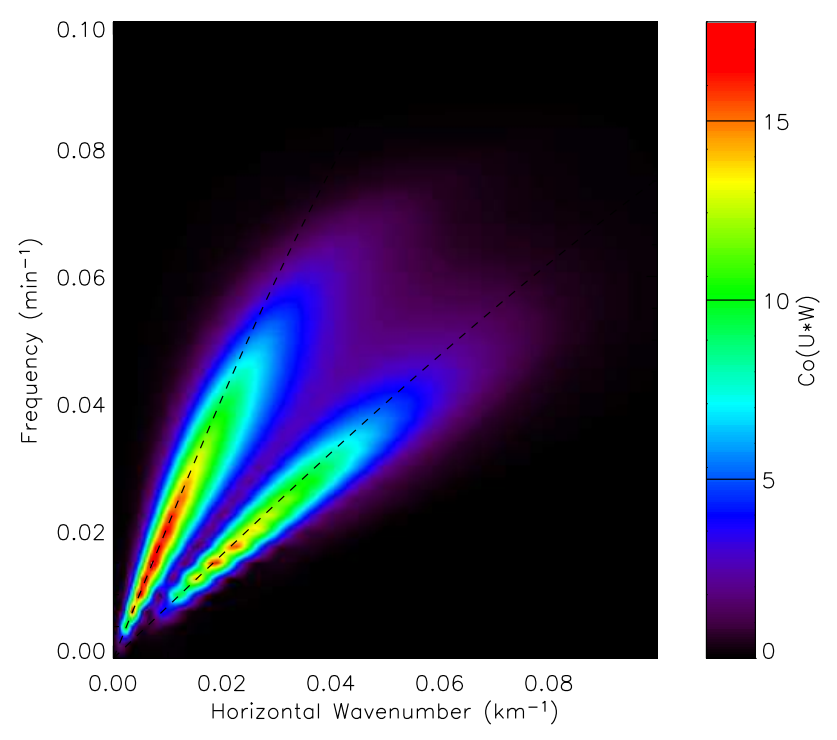

Fig. 8. Two-dimensional $(k, \omega)$ Fourier cospectrum of $u^{\prime}$ and $w^{\prime}$. The dashed lines are lines of constant vertical wavelength $L_{z}=4.3 \mathrm{~km}$ (shallower slope) and $L_{z}=11 \mathrm{~km}$ (steeper slope). Note that units for wavenumber and frequency are cycles $/ \mathrm{km}$ and cycles/min, respectively, in this and subsequent plots.

in Fig. 1a is expanded into a Fourier sine series in the domain $0 \leq z \leq 150 \mathrm{~km}$, there is a strong response for halfwavelengths greater than $10 \mathrm{~km}$ and a secondary maximum at about $4.4 \mathrm{~km}$ (recall that it is the half-wavelength of the tropospheric source that corresponds to the wavelength of the stratospheric response). It is also notable that although the depth of the heating layer at any instant is $6 \mathrm{~km}$, the vertical wavelength spectrum of the time average heating spectrum actually has a minimum at $6 \mathrm{~km}$. The response will of course depend on the imposed heating, and a response dominated by two distinct vertical wavelengths would not be expected for any arbitrary vertical distribution of the heat source.

Figure 9 shows the results of the Fourier cospectral analysis in $(x, z)$ within the domain $14.25<z<75.25 \mathrm{~km}$ and $0<x<1150.5 \mathrm{~km}$ for four different 1 -h periods. These show the time evolution of the momentum flux in the wave field. The packet that appears first is characterized by longer $L_{z}$ (smaller $m=1 / L_{z}$ ), which carries the largest momentum fluxes. At later times, some of the longest $L_{z}$ waves with shorter $L_{x}=1 / k$ have left the domain. The slower moving shorter $L_{z}$ waves with longer $L_{x}$ remain, and the much shorter $L_{z}=4.3 \mathrm{~km}$ waves have propagated into the stratosphere with sufficient amplitude to become apparent. At hour 6 , two distinct packets remain that have rather distinct distributions in $(k, m)$. The weaker momentum flux in the $L_{z}=4.3 \mathrm{~km}$ packet is apparent from Fig. 9 .

Note that in Fig. 8, the two wave packets appear to have more comparable cospectral power. The apparent discrepancy is related to the averaging timescale in these two figures. The $L_{z}=4.3 \mathrm{~km}$ packet has slower vertical group speed and is less dispersive than the longer $L_{z}$ wave packet. The longest $L_{z}$ waves propagate through the domain relatively quickly. So the flux associated with these is large in the 1$\mathrm{h}$ time average early in the simulation (Fig. 9) but appears weaker, more comparable in magnitude to the short vertical wavelength packet, if the fluxes are averaged over $8 \mathrm{~h}$ (Fig. 8).

\section{Implications for observing systems}

The cross-sections of disturbance vertical motion shown in Fig. 2 suggest that the character of the locally observed response to a convective heat source depends strongly on the distance of the observer from the source. In order to demonstrate this point in a more quantitative fashion, we sample the wave field as if observed by imaginary profiler instruments located at four "sites" at distances of 25, 50, 100, and $200 \mathrm{~km}$ from the source. For best comparison to the results presented in the previous sections, our imaginary profilers can measure both $u^{\prime}$ and $w^{\prime}$ as functions of height and time, at the same resolution, and over the same altitude range and time period as in Sect. 5. However, no horizontal $x$ information is obtained from any of the imaginary profilers' measurements.

The temporal evolution of the vertical wavelength Fourier spectra computed at each profiler site is shown in Fig. 10. The signal seen by an observer at a given distance from the source is initially dominated by long vertical wavelength waves associated with the fast moving wave-packet, and then later by shorter vertical wavelengths associated with the slower packet. The characteristics of the waves observed is a strong function of distance from the source. Close to the source $(x=25 \mathrm{~km})$ the waves are only observed for a short time, and the stratospheric response is dominated by the 

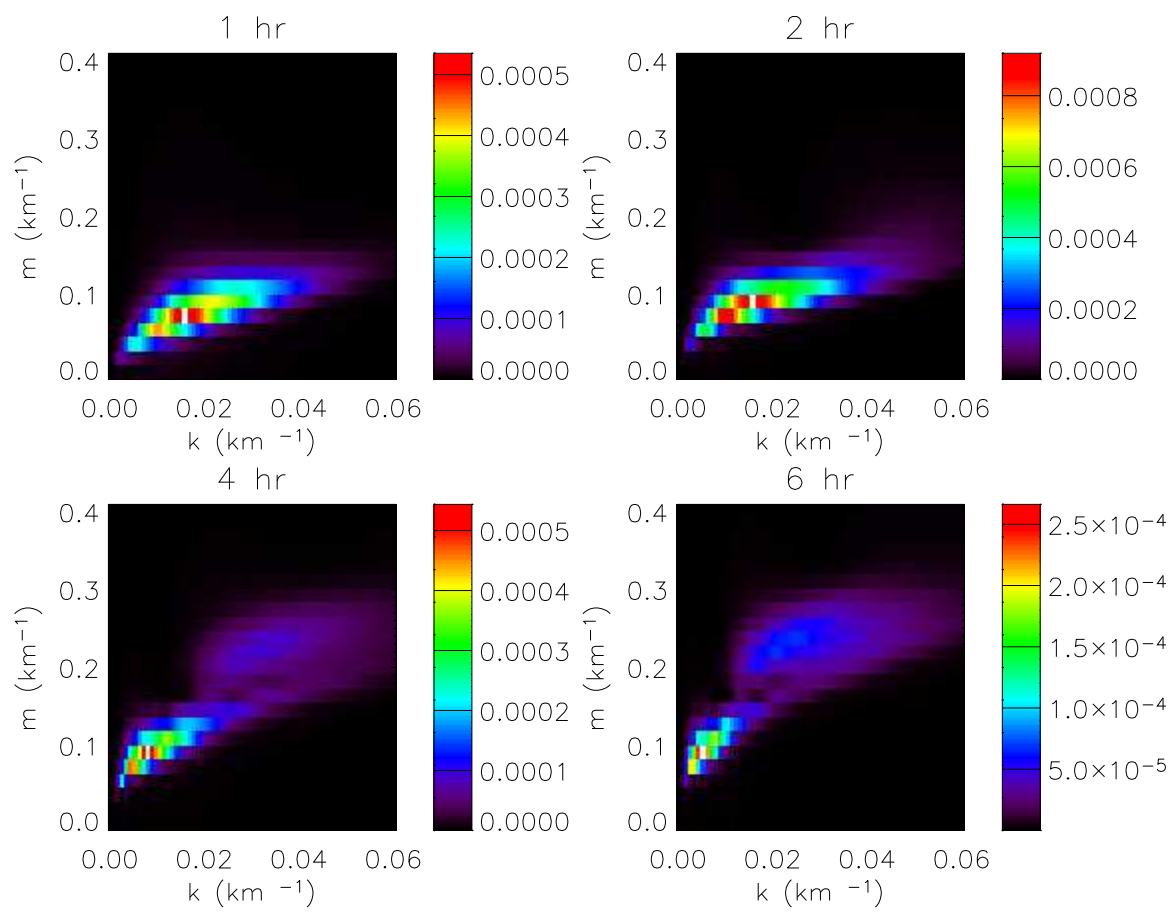

Fig. 9. Two-dimensional $(k, m)$ Fourier cospectra of $u^{\prime}$ and $w^{\prime}$ averaged over 1-h periods centered on hours 1, 2, 4, and 6 .

long wavelength packet since the short vertical wavelength waves have slower vertical group velocity and do not reach the stratosphere until they have propagated further from the source. Some of the longest vertical wavelength waves are observed only at the closest site. At larger horizontal distances from the source, two wave packets are clearly observed, arriving at different times. For these waves horizontal wavelength greatly exceeds vertical wavelength, so that the hydrostatic approximation holds. Furthermore, the vertical wavenumber increases linearly with time, reflecting the fact noted below Eq. (15) that for hydrostatic waves $c_{p x} \simeq N L_{z} /(2 \pi)$, so that the vertical wavenumber, $m$, approximately satisfies the relation $m \simeq N / c_{p x}=N \delta t / \delta x$, where $\delta x$ is the horizontal distance traveled by a wave in the time interval $\delta t$. Thus, at a given horizontal location $m$ increases linearly in time.

To elucidate the frequency content of the two wave packets, a Morlet wavelet analysis (Press et al., 1992) has been performed on the data in the time domain. The wavelet analysis produces similar results to the Fourier analysis, but with some loss of spectral resolution. Wavelet analysis can, however, resolve the spectral properties of each wave-packet as functions of time and/or height for differing horizontal locations. Also, unlike Fourier and discrete wavelet transforms, continuous wavelet transforms such as the Morlet are not energy conserving, so we have normalized each transform with the total covariance in the time series, such that $\int u^{\prime} w^{\prime} d t=\int \operatorname{Co}(U \cdot W) d \omega$.
Figure 11 shows the wavelet cospectra for the time series sampled at the four profiler sites and averaged in $z$. At $x=25 \mathrm{~km}$ only a single wave packet has significant amplitude. This packet appears within the first hour and is associated with the longer $L_{z}$ waves. As distance from the source increases the dominant wave period increases. The waves appear in two separate packets arriving at different times, consistent with the Fourier analysis results. Note, however, that at any one site the two wave packets contain the same dominant frequency.

This result can be understood by noting that the ratio of wave frequency to buoyancy frequency determines the angle to the vertical at which waves propagate. Thus, as distance from the source increases the frequency of the waves observed in the stratosphere must accordingly decrease. Figure 12 schematically illustrates the wave field and the profiler measurements at the four sites. Lines of constant phase correspond roughly to group propagation rays whose slope will depend on frequency as $\omega / N=\cos \theta$, where $\theta$ is the angle from the vertical. The range of frequencies that will be visible at each site will correspond to the steepest and shallowest rays that intersect the profile at the top and bottom of the observation height range. Note that this range of frequencies will be different at each site. The range will also be narrower for more limited observation height ranges. These results underscore the fact that the location of a site and the observation height range can have a controlling influence on the properties of the waves observed if the sources also tend 

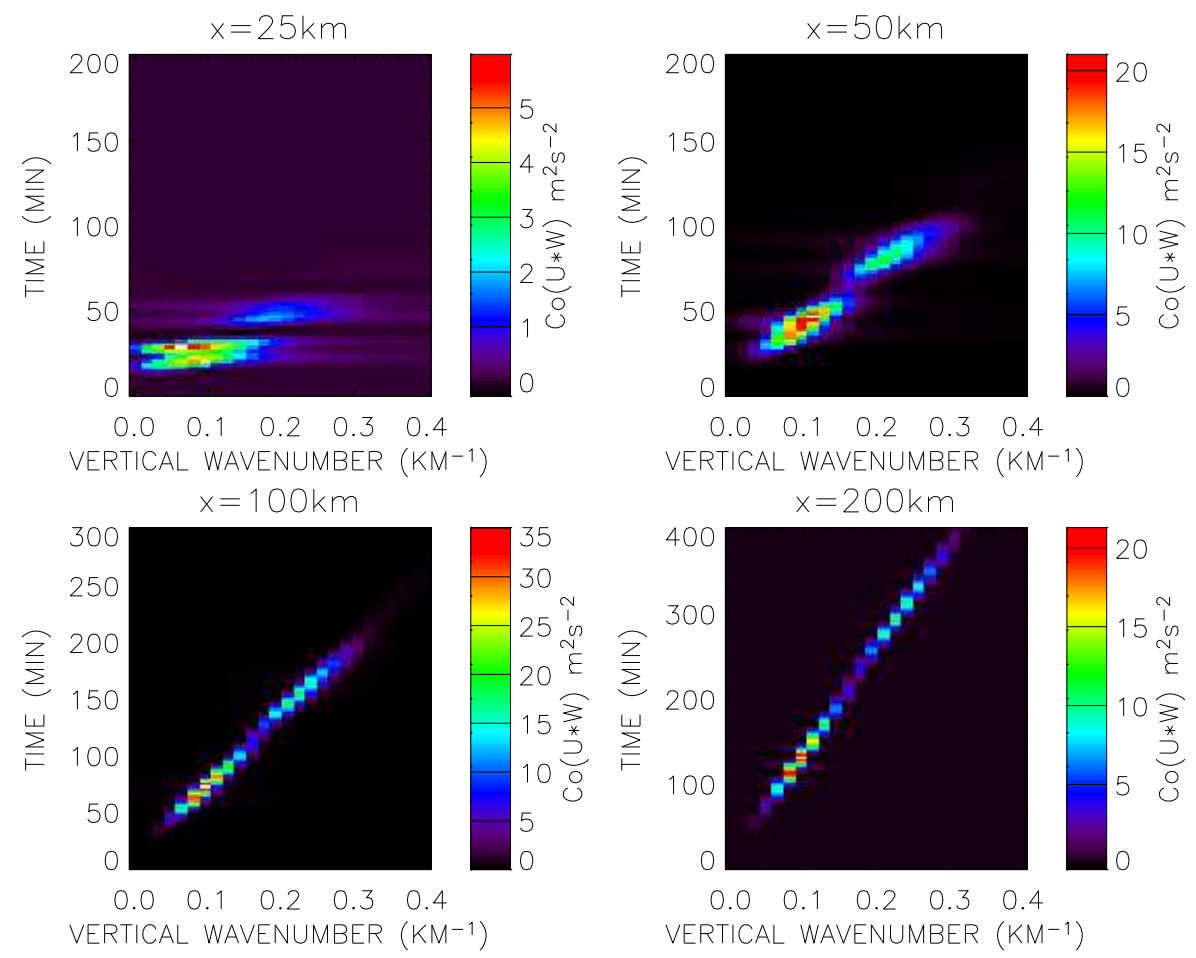

Fig. 10. One-dimensional Fourier cospectra of $u^{\prime}$ and $w^{\prime}$ as functions of vertical wavenumber and time for each of the four sites.
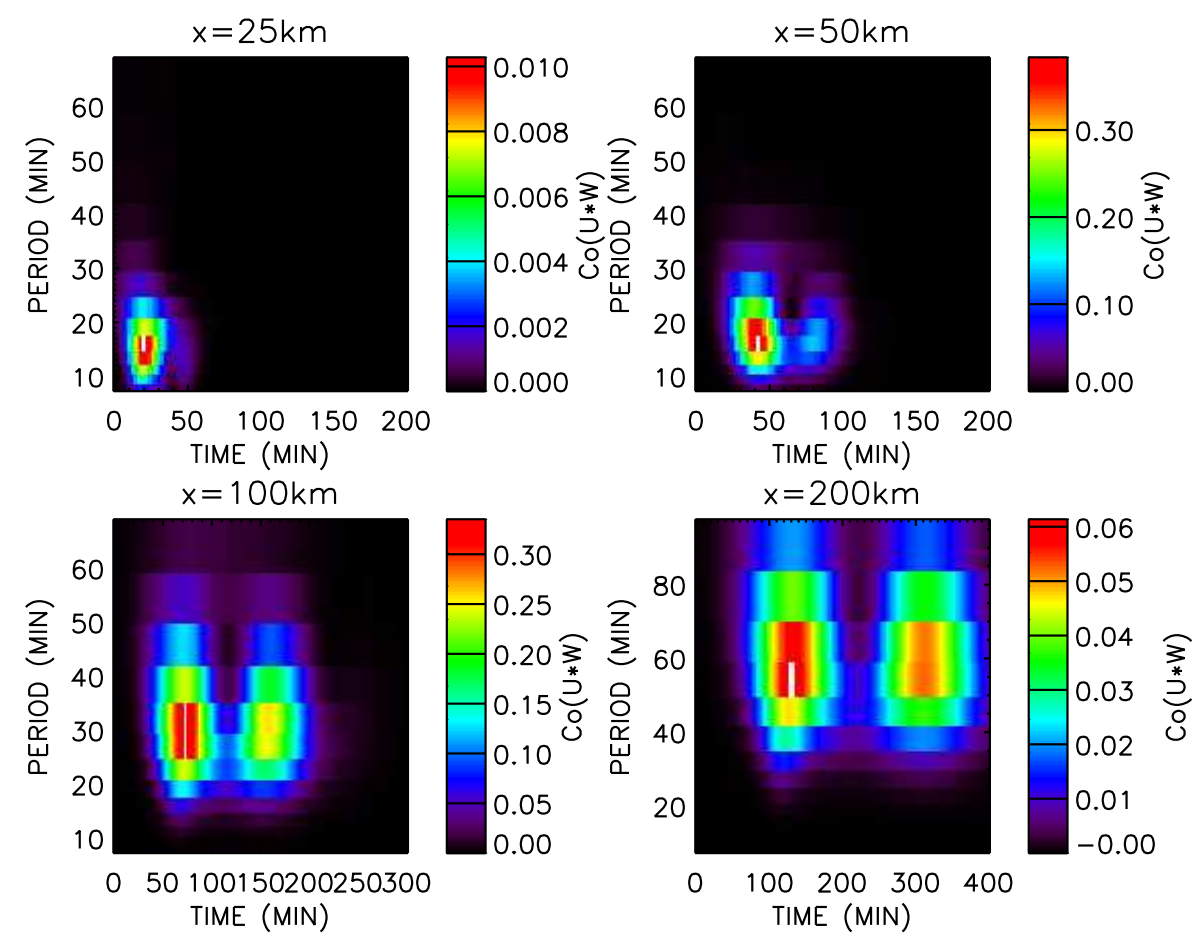

Fig. 11. Morlet wavelet cospectra of $w^{\prime}$ and $u^{\prime}$ as functions of time and frequency $\omega$ at each of the four profiler sites. 
to be fixed to certain geographic locations. Likewise, the timing of the observations can also have a controlling influence on the wave properties observed.

\section{Conclusions}

This work has demonstrated that transient localized heat sources can generate waves with very long vertical wavelengths and high phase speeds. These waves can readily propagate into the upper atmosphere. The wave field from the simple transient source used in the model described here displays a complex array of wavelengths and frequencies. Dispersion of the waves leads to distinct packets of wave activity with different properties. The dominant mode observed at any given location depends strongly on the position of the observer from the source. As shown in Figs. 1012 , an observer close to the source would see long vertical wavelength waves with high frequencies lasting for a short time, while further from the source the dominant vertical wavelength would be smaller and frequency lower, the waves would arrive later, and they would persist longer. Also, some of the very longest vertical wavelength waves can only be observed very close to the source and will be missed entirely by observers outside of a limited range.

From the above analysis it is clear that the wave components with largest vertical group velocity quickly move upward into the strongly damped region above $100 \mathrm{~km}$ and can only be sampled in the stratosphere for a very short period of time. Furthermore, since such waves have long vertical wavelengths, observational techniques that rely on finding short vertical wavelength features in individual profiles of temperature (for example as from radiosondes) cannot be used to reliably estimate their contribution to upper atmospheric wave driving. Again using the radiosonde example, twice daily soundings may also fail entirely to see all but the lowest frequency waves if the timing and location of the observation is not in the right range. Similar issues of measurement timing and location will have important effects on results of analyses of satellite based observations.

The short vertical wavelength, and low vertical group velocity components of the spectrum, on the other hand, are much more likely to be observed in the stratosphere, and hence may appear to dominate gravity wave momentum fluxes in observational studies which cannot resolve long vertical wavelength features (Alexander et al., 2002).

These results illustrate how intermittency in gravity wave sources can influence observations. The observational technique limitations, the timing, and the geographic locations must all be considered in the interpretation of any data set. It is also clear that a combination of techniques is required to properly sample the stratospheric wave spectrum generated by convective gravity wave sources.

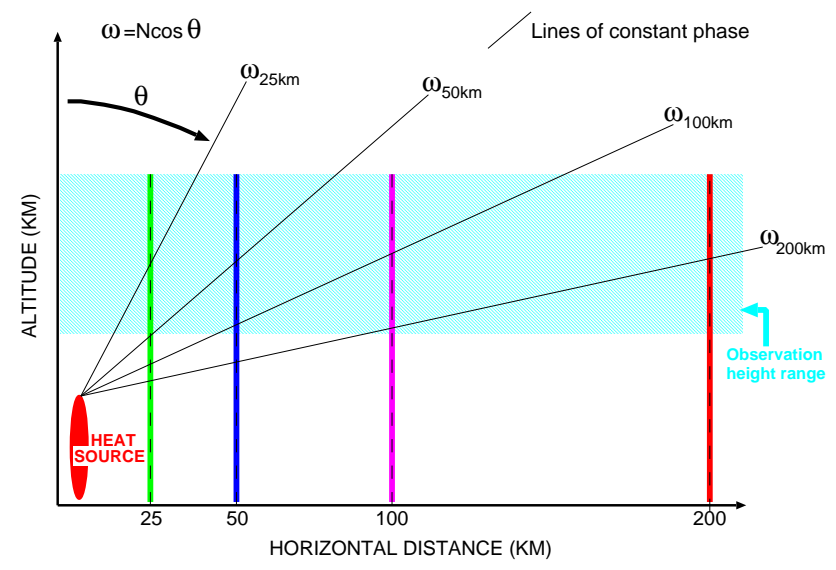

Fig. 12. Schematic of the wave field observed at the four observation sites denoted by the four colored vertical lines. The range of frequencies observed at each site will correspond roughly to frequencies associated with the steepest and shallowest lines of constant phase that intersect the top and bottom of the observation height range (blue shading).

Acknowledgements. This work was supported by the National Science Foundation Division of Atmospheric Sciences via grants ATM-0225441 and ATM-0234230.

Edited by: W. Ward

\section{References}

Alexander, M. J.: Interpretations of observed climatological patterns in stratospheric gravity wave variance, J. Geophys. Res. 103, 8627-8640, 1998

Alexander, M. J. and Holton, J. R.: A model study of zonal forcing in the equatorial stratosphere by convectively induced gravity waves, J. Atmos. Sci., 54, 408-419, 1997.

Alexander, M. J., Tsuda, T., and Vincent, R. A.: On the latitudinal variations observed in gravity waves with short vertical wavelengths, J. Atmos. Sci., 59, 1394-1404, 2002.

Beres, J. H., Alexander, M. J., and Holton, J. R.: Effects of tropospheric wind shear on the spectrum of convectively generated gravity waves, J. Atmos. Sci., 59, 1805-1824, 2002.

Bretherton, C.: Group velocity and the linear response of stratified fluids to internal heat or mass sources, J. Atmos. Sci., 45, 81-93, 1988.

Fritts, D. C. and Alexander, M. J.: A review of gravity wave dynamics and effects on the middle atmosphere, Rev. Geophys., 41, doi:10.1029/2001RG000 106, 2003.

Fritts, D. C. and Vincent, R. A.: Mesospheric momentum flux studies at Adelaide, Australia: observations and a gravity wave-tidal interaction model, J. Atmos. Sci., 44, 605-619, 1987.

Holton, J., Beres, J., and Zhou, X.: On the vertical scale of gravity waves excited by localized thermal forcing, J. Atmos. Sci., 59, 2019-2023, 2002

Mapes, B. E.: Gregarious tropical convection, J. Atmos. Sci., 50, 2026-2037, 1993 
McLandress, C., Alexander, M. J., and Wu, D.: Microwave Limb Sounder observations of gravity waves in the stratosphere: A climatology and interpretation, J. Geophys. Res., 105, $11947-$ 11 967, 2000.

Nicholls, M., Pielke, R., and Cotton, W.: Thermally forced gravity waves in an atmosphere at rest, J. Atmos. Sci., 48, 1869-1884, 1991.

Pandya, R. E. and Alexander, M. J.: Linear stratospheric gravity waves above convective thermal forcing, J. Atmos. Sci., 56, 2434-2446, 1999.
Press, W., Flannery, B., Teukolsky, S., and Vetterling, W.: Numerical Recipes, Cambridge Univ. Press, Cambridge, 1992.

Salby, M. L. and Garcia, R. R.: Transient Response to localized Episodic Heating in the Tropics. Part I: Excitation and ShortTime Near-Field Behaviour, J. Atmos. Sci., 44, 458-498, 1987.

Song, I.-S., Chun, H.-Y., and Lane, T. P.: Generation mechanisms of convectively forced internal gravity waves and their propagation to the stratosphere, J. Atmos. Sci., 60, 1960-1980, 2003. 